\title{
BMJ Open Living experiences of patients with advanced cancer with low socioeconomic status: protocol for a systematic review of qualitative evidence
}

\author{
Zifen An (D) , Xianmei Meng (D) , Pei Fang (D) , Huidan Yu (D) , Liping Yu
}

To cite: An Z, Meng X,

Fang $P$, et al. Living experiences of patients with advanced cancer with low socioeconomic status: protocol for a systematic review of qualitative evidence. BMJ Open 2022;12:e054606. doi:10.1136/ bmjopen-2021-054606

- Prepublication history and additional supplemental material for this paper are available online. To view these files, please visit the journal online (http://dx.doi.org/10.1136/ bmjopen-2021-054606).

$\mathrm{ZA}$ and $\mathrm{XM}$ contributed equally.

$\mathrm{ZA}$ and $\mathrm{XM}$ are joint first authors.

Received 17 June 2021

Accepted 12 January 2022

Check for updates

(c) Author(s) (or their employer(s)) 2022. Re-use permitted under CC BY-NC. No commercial re-use. See rights and permissions. Published by BMJ.

School of Nursing, Wuhan University, Wuhan, Hubei, China

Correspondence to

Professor Liping Yu; yuliping@whu.edu.cn and

Dr Huidan Yu;

yuhuidan32@126.com

\section{ABSTRACT}

Introduction The number of patients with advanced cancer is rapidly increasing, and the disease burden among those with low socioeconomic status (SES) has accordingly become a global concern. Low SES can adversely impact patients with advanced cancer. The purpose of this systematic review is to shed light on the life experiences of patients with advanced cancer with low SES to help provide targeted and effective strategies to improve their quality of life.

Methods and analysis We will include the following English databases: Cochrane Library, Cumulative Index to Nursing and Allied Health Literature, PubMed, MEDLINE, Embase, Web of Science, Joanna Briggs Institute (JBI) Database of Systematic Reviews, PsycINF0 and OpenGrey, and the following Chinese databases: China National Knowledge Infrastructure, VIP Database for Chinese Technical Periodicals and Wanfang Data Knowledge Service Platform. A comprehensive search of qualitative studies on the experiences of patients with advanced cancer with low SES will be conducted from the above databases, with no age limit. Quality assessments of the studies will be independently performed by two reviewers using the JBI Critical Assessment Checklist, and any disagreements will be resolved through a discussion with a third reviewer. Relevant data will be extracted using the JBI standardised data extraction tools. The JBI meta-aggregation tool will be used to compare, analyse and summarise the original results. The reliability and credibility of the overall quality of the studies included will be evaluated using the JBI ConQual approach.

Ethics and dissemination This study is based on existing public literature and therefore does not require a formal ethics review. The results of the study may be presented in peer-reviewed international journals and presented at scientific conferences.

PROSPERO registration number CRD42021250423.

\section{INTRODUCTION}

Cancer remains the leading cause of death worldwide, and an estimated 10 million cancer deaths occurred in $2020 .^{1}$ The global burden of cancer-related diseases is also increasing. ${ }^{2}$ Patients with advanced cancer are those with metastatic or controlled but incurable cancer. ${ }^{3}$ Although novel treatment
Strengths and limitations of this study

- There is an urgent need for qualitative evidence regarding the life experiences of patients with advanced cancer with low socioeconomic status (SES) to help formulate appropriate interventions.

- This study is the first qualitative systematic review to focus on the living experiences of patients with advanced cancer with low SES.

- This study will include a systematic review of empirical evidence based on qualitative research conducted across multiple regions and cultures that will contribute to the dissemination of care practices for patients with advanced cancer with low SES.

- The findings of this qualitative systematic review are limited by the context and background of the included original studies.

modalities and the quality of care strategies have improved the overall 5-year survival rate of patients with advanced cancer, ${ }^{45}$ no curative cancer modality has been developed. ${ }^{6}$ Patients with advanced cancer experience adverse health outcomes, and majority do not recover. ${ }^{7}$ Within the limited survival period of patients with advanced cancer, the longterm consequences of cancer and its treatment often result in higher symptom loads, ${ }^{89}$ including moderate to severe cancer pain, ${ }^{10}$ depression, ${ }^{11}$ malnutrition ${ }^{12}$ and cancerrelated fatigue. ${ }^{13}$ These, in turn, result in a significantly decreased quality of life,${ }^{14}$ with severely impaired overall physical, psychological, and social functions and a higher risk of suicidal intentions. ${ }^{15}$

Patients with advanced cancer with low socioeconomic status (SES), that is, those generally either with residence in a deprived regional status or with low income, ${ }^{16}$ face more complex problems. ${ }^{17}$ Despite their varying types of cancer, patients with advanced cancer with low SES have similar concerns and issues. They often experience 
delays in perceiving non-specific symptoms of certain cancers (eg, fatigue or unexplained weight loss) until the time of diagnosis. ${ }^{18}{ }^{19}$ Further, active clinical treatment is often associated with higher out-of-pocket costs in these patients than their high SES counterparts. ${ }^{20-22}$ A systematic review by Iragorri $e t a l^{21}$ showed that patients with cancer residing in low-income areas spent $42 \%$ of their annual income on cancer-related out-of-pocket expenses. This was approximately 2.6 times higher than the out-ofpocket expense-to-annual income ratio for patients with cancer in high-income areas. Moreover, the debilitating effects of late illness often lead to unemployment for both patients and their caregivers, ${ }^{23}{ }^{24}$ further lowering the total household income. In addition, the economic cost of advanced cancer treatment is only partially covered by the social security system. ${ }^{22}$

The negative impact of long-term and costly treatment and low income on the patients' quality of life is often multidimensional. ${ }^{25}$ Some studies have shown patients' higher drug non-compliance to save costs (eg, reducing drug use, delaying prescriptions, using alternative therapies) in patients with advanced cancer with low SES. ${ }^{26}{ }^{27}$ Further, these patients are forced to interrupt or abandon treatment. ${ }^{28}{ }^{29}$ Lower treatment compliance can have significantly negative health-related consequences, including increased hospitalisations ${ }^{30}$ and higher mortality rates. ${ }^{17}$ A large National Health Survey found that patients who reported having financial problems because of cancer care costs were not only more likely to report lower health conditions, but also had worse mental health status. ${ }^{31}$ Severe symptoms of anxiety and depression lead to poorer quality of life, ${ }^{32}{ }^{33}$ which increases the need for palliative care. ${ }^{34}$

Despite these adverse effects of low SES, the care plan for patients with advanced cancer with low SES has not been clearly defined. Only a few quantitative studies have explored effective nursing strategies for patients with advanced cancer, including symptom management ${ }^{35}$ and psychosocial care. ${ }^{36}{ }^{37}$ However, these methods often do not meet the daily care needs of patients with advanced cancer with low SES. ${ }^{38}$ For example, symptom management is continuous and dynamic, and regular medication use is better for symptom control. However, patients with advanced cancer with low SES often adjust or delay medication due to their limited financial resources. ${ }^{262739}$ These patients also often lack access to adequate and continuous psychosocial care services because of socioeconomic restrictions. ${ }^{40}$ Some qualitative studies have found more life difficulties in patients with advanced cancer with low SES. Van Roij et al reported that patients in their study felt overwhelmed but were embarrassed when seeking financial support. ${ }^{25}$ These patients also often experience stronger feelings of social exclusion and isolation than their high SES counterparts. ${ }^{25}$ In addition, their strategies for accepting and managing behavioural changes under such economic hardship may be unique. ${ }^{41}$ For instance, the more effective strategies of pain management among these patients were found as the sensory experience of pain and the meaning of pain, rather than prescribed analgesics. ${ }^{39}$

Therefore, this qualitative review aims to shed light on the life experiences of patients with advanced cancer with low SES, in a detailed manner, including disease distress, barriers and strategies in coping with the disease distress. Ultimately, the synthesised qualitative evidence helps provide targeted and appropriate care strategies to improve patients' quality of life.

\section{METHODS AND ANALYSIS}

This is a qualitative systematic review protocol that follows the Preferred Reporting Items for Systematic Reviews and Meta-analysis Protocols checklist to ensure that the research plan is robust (online supplemental material 1).

\section{Inclusion criteria}

Participants

We will review all studies that include patients with advanced cancer, without limitations on the country or type of cancer.

\section{Phenomenon of interest}

This review will include studies that describe the life experiences of patients with advanced cancer, including disease distress, barriers and strategies in detail.

\section{Context}

The context will consider the life experiences of patients with advanced cancer with low SES. According to literature review, most previous studies identified low income as a feature of low SES. ${ }^{16} 1719$ Therefore, low income will be considered as low SES in this study. Also, due to the varying standards of low income in different locations, patients with advanced cancer who are identified as having a low-income economic status in the original research will be included in this study.

\section{Types of studies}

The review will consider qualitative studies, including, but not limited to, personal narratives, grounded theories, ethnographies and feminist research. Only English and Chinese literature will be included, and there will be no restrictions on the year of publication.

\section{Patient and public involvement}

No patient will be involved in the design, planning and conception of this study.

\section{Search strategy}

The search strategy aims to find both published and grey literature. An initial search will be conducted using the PubMed and Cumulative Index to Nursing and Allied Health Literature (CINAHL) databases. This will be followed by an analysis of MeSH (Medical Subject Headings) terminologies included in the title and abstract and index terminology terms used to describe the articles. A comprehensive search will also be performed, using 


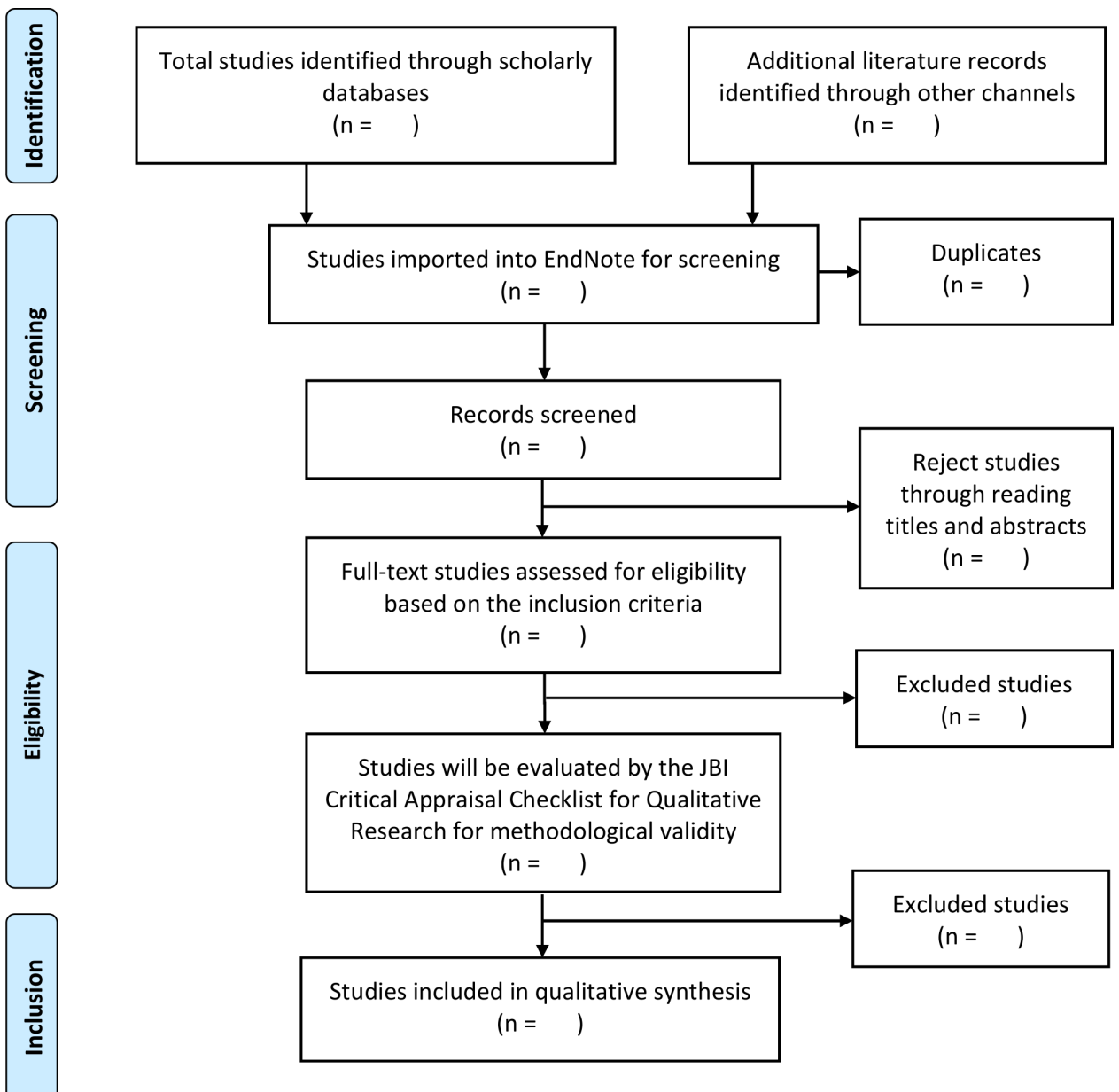

Figure 1 PRISMA flow diagram of the study process. JBI, Joanna Briggs Institute; PRISMA, Preferred Reporting Items for Systematic Reviews and Meta-Analysis.

the relevant $\mathrm{MeSH}$ terminology and index terminology terms, in the following databases: the Cochrane Library, CINAHL, PubMed, MEDLINE, Embase, Web of Science, Joanna Briggs Institute (JBI) Database of Systematic Reviews, PsycINFO, China National Knowledge Infrastructure, VIP Database for Chinese Technical Periodicals, Wanfang Data Knowledge Service Platform and OpenGrey. The complete search strategy for the customisations used in PubMed is presented in online supplemental material 2.

\section{Study selection}

All the identified studies will be collated and uploaded to EndNote V.X9 software; duplicate studies will be eliminated. Two independent reviewers (ZA and XM) will screen the titles and abstracts according to the inclusion criteria. Articles that do not meet the inclusion criteria will be excluded, and the reason for exclusion will be attached as supplemental material in the final systematic review report. To maintain the credibility of the screening process, all included studies will be screened according to a rigorous process, and any disagreements will be resolved through discussion with a third reviewer (HY).

\section{Assessment of methodological quality}

Quality assessments prior to inclusion in the review will be performed by two independent reviewers (ZA and XM) according to the 10-item checklist of the JBI Qualitative Assessment and Review Instrument for methodological validity. ${ }^{42}$ The checklist assesses different domains, including research methodology, philosophical foundation, data collection, analysis method and result validity (online supplemental material 3). All studies will be evaluated based on whether or not the study being evaluated fulfils the checklist item for each domain. Items in the checklist will be marked as 'yes' if the study fulfils the domain criteria, 'no' if it does not and 'unclear' if the study's adherence to certain domain criteria cannot be conclusively proven. The result of the evaluation will be determined based on the number of domain items (of a total of 10) that the study fulfils, with a rating of $\leq 6$ considered weak, $7-8$ considered moderate and 9-10 considered high quality. Any disagreements will be resolved through a discussion with the third reviewer (HY) until a consensus is reached. For studies that are evaluated as moderate and above, data will be extracted and integrated for analysis. 


\section{Data collection}

Two independent reviewers (ZA and XM) will collect qualitative data related to the research questions and objectives using the JBI qualitative assessment and review instrument from the JBI System for the Unified Management, Assessment and Review of Information. ${ }^{43}$ The extracted data will include details regarding the populations, contexts, methods, culture, geographical location, study methods and the phenomena of interest (online supplemental material 4). All information obtained will be grouped into tables.

\section{Data synthesis}

The collected data will be organised and synthesised using the JBI meta-aggregation method.$^{44}$ Before integration, two independent reviewers (ZA and XM) will read the articles to understand the full text. They will then summarise the quality of the results and divide them into three levels: unequivocal, equivocal and unsupported. These results will then be further classified to arrive at a set of meaningful concepts that are consistent with those of the original manuscript. These similar categories are eventually integrated to produce a comprehensive set of findings that can improve the living experiences of patients with advanced cancer with low SES.

\section{Assessing the accuracy of results}

Ultimately, the accuracy of the findings will be evaluated based on the JBI ConQual approach, ${ }^{45}$ which evaluates the reliability and credibility of the findings. The confidence level of the final study results will be classified into four scales of high, moderate, low or very low (online supplemental material 5). The process will be completed by two independent reviewers (ZA and XM), and any disagreement will be resolved through a discussion. The entire protocol process is illustrated in figure 1 .

\section{Reporting of protocol}

The findings of the comprehensive review in this qualitative study will be reported in accordance with the Enhancing Transparency in Reporting the Synthesis of Qualitative Research ${ }^{46}$ guideline to ensure that the review and research is robust (online supplemental material 6).

\section{ETHICS AND DISSEMINATION}

This systematic review will identify and integrate the life experiences of patients with advanced cancer with low SES to understand the other issues and needs of such a vulnerable population, apart from financial barriers, to provide targeted care to improve patients' quality of life. The findings will be published in a peer-reviewed journal or presented at scientific conferences.

Contributors ZA and XM contributed equally to this work. ZA and XM contributed to the conception and design of the study. The introduction was written by ZA and $\mathrm{XM}$. The methods, including literature retrieval, data extraction and appraisal, risk offset assessment and data synthesis, were drafted by HY and LY. In addition, PF, LY and $\mathrm{HY}$ assisted in clarifying differences to avoid errors. All authors contributed to the final manuscript and agreed with its publication.
Funding This work was supported by the Health Commission of Hubei Province Scientific Research Project (grant number WJ2019M176).

Competing interests None declared.

Patient consent for publication Not required.

Provenance and peer review Not commissioned; externally peer reviewed.

Supplemental material This content has been supplied by the author(s). It has not been vetted by BMJ Publishing Group Limited (BMJ) and may not have been peer-reviewed. Any opinions or recommendations discussed are solely those of the author(s) and are not endorsed by BMJ. BMJ disclaims all liability and responsibility arising from any reliance placed on the content. Where the content includes any translated material, BMJ does not warrant the accuracy and reliability of the translations (including but not limited to local regulations, clinical guidelines, terminology, drug names and drug dosages), and is not responsible for any error and/or omissions arising from translation and adaptation or otherwise.

Open access This is an open access article distributed in accordance with the Creative Commons Attribution Non Commercial (CC BY-NC 4.0) license, which permits others to distribute, remix, adapt, build upon this work non-commercially, and license their derivative works on different terms, provided the original work is properly cited, appropriate credit is given, any changes made indicated, and the use is non-commercial. See: http://creativecommons.org/licenses/by-nc/4.0/.

\section{ORCID iDs}

Zifen An http://orcid.org/0000-0002-1624-8153

Xianmei Meng http://orcid.org/0000-0003-0036-2733

Pei Fang http://orcid.org/0000-0003-1922-4955

Huidan Yu http://orcid.org/0000-0002-5353-3025

Liping Yu http://orcid.org/0000-0002-3202-3472

\section{REFERENCES}

1 Sung H, Ferlay J, Siegel RL, et al. Global cancer statistics 2020: GLOBOCAN estimates of incidence and mortality worldwide for 36 cancers in 185 countries. CA Cancer J Clin 2021;71:209-49.

2 Sullivan R, Peppercorn J, Sikora K, et al. Delivering affordable cancer care in high-income countries. Lancet Oncol 2011;12:933-80.

3 Cancer AJCO. American Cancer Society. What is advanced cancer?, 2020. Available: https://www.cancer.org [Accessed 10 Sep 2020].

4 Allemani C, Weir HK, Carreira H, et al. Global surveillance of cancer survival 1995-2009: analysis of individual data for $25,676,887$ patients from 279 population-based registries in 67 countries (CONCORD-2). Lancet 2015;385:977-1010.

5 Allemani C, Matsuda T, Di Carlo V, et al. Global surveillance of trends in cancer survival 2000-14 (CONCORD-3): analysis of individual records for 37513025 patients diagnosed with one of 18 cancers from 322 population-based registries in 71 countries. Lancet 2018;391:1023-75.

6 Wang J-J, Lei K-F, Han F. Tumor microenvironment: recent advances in various cancer treatments. Eur Rev Med Pharmacol Sci 2018;22:3855-64.

7 Foreman KJ, Marquez N, Dolgert A, et al. Forecasting life expectancy, years of life lost, and all-cause and cause-specific mortality for 250 causes of death: reference and alternative scenarios for 2016-40 for 195 countries and territories. Lancet 2018;392:2052-90.

8 Kuon J, Vogt J, Mehnert A, et al. Symptoms and needs of patients with advanced lung cancer: early prevalence assessment. Oncol Res Treat 2019;42:650-9.

9 Seow H, Barbera L, Sutradhar R, et al. Trajectory of performance status and symptom scores for patients with cancer during the last six months of life. J Clin Oncol 2011;29:1151-8.

10 van den Beuken-van Everdingen MHJ, Hochstenbach LMJ, Joosten EAJ, et al. Update on prevalence of pain in patients with cancer: systematic review and meta-analysis. J Pain Symptom Manage 2016;51:1070-90.

11 Okuyama T, Akechi T, Mackenzie L, et al. Psychotherapy for depression among advanced, incurable cancer patients: a systematic review and meta-analysis. Cancer Treat Rev 2017;56:16-27.

12 Ravasco P, Monteiro-Grillo I, Vidal PM, et al. Nutritional deterioration in cancer: the role of disease and diet. Clin Oncol 2003;15:443-50.

13 Mohandas H, Jaganathan SK, Mani MP, et al. Cancer-related fatigue treatment: an overview. J Cancer Res Ther 2017;13:916-29.

14 Ahlam A, Hind M, Haddou Rahou B, et al. Quality of life of Moroccan patients on the palliative phase of advanced cancer. BMC Res Notes 2019;12:351. 
15 McFarland DC, Walsh L, Napolitano S. Suicide in patients with cancer: identifying the risk factors. Oncology 2019;33:221-6.

16 Jang B-S, Chang JH. Socioeconomic status and survival outcomes in elderly cancer patients: a national health insurance service-elderly sample cohort study. Cancer Med 2019;8:3604-13.

17 Wang N, Cao F, Liu F, et al. The effect of socioeconomic status on health-care delay and treatment of esophageal cancer. J Trans/ Med 2015;13:241.

18 Marcu A, Black G, Vedsted P, et al. Educational differences in responses to breast cancer symptoms: a qualitative comparative study. Br J Health Psychol 2017;22:26-41.

19 McCutchan GM, Wood F, Edwards A, et al. Influences of cancer symptom knowledge, beliefs and barriers on cancer symptom presentation in relation to socioeconomic deprivation: a systematic review. BMC Cancer 2015;15:1000.

20 Zafar SY. Financial toxicity of cancer care: it's time to intervene. $J$ Natl Cancer Inst 2016;108. doi:10.1093/jnci/djv370. [Epub ahead of print: 1112 2015].

21 Iragorri N, de Oliveira C, Fitzgerald N, et al. The out-of-pocket cost burden of cancer care-A systematic literature review. Curr Oncol 2021;28:1216-48.

22 Kankeu HT, Saksena P, Xu K, et al. The financial burden from noncommunicable diseases in low- and middle-income countries: a literature review. Health Res Policy Syst 2013;11:31.

23 Mols F, Tomalin B, Pearce A, et al. Financial toxicity and employment status in cancer survivors. A systematic literature review. Support Care Cancer 2020;28:5693-708.

24 Azzani M, Roslani AC, Su TT. The perceived cancer-related financial hardship among patients and their families: a systematic review. Support Care Cancer 2015;23:889-98.

25 van Roij J, Brom L, Youssef-El Soud M, et al. Social consequences of advanced cancer in patients and their informal caregivers: a qualitative study. Support Care Cancer 2019;27:1187-95.

26 Kaul S, Avila JC, Mehta HB, et al. Cost-related medication nonadherence among adolescent and young adult cancer survivors. Cancer 2017;123:2726-34.

27 Chung GC, Marottoli RA, Cooney LM, et al. Cost-related medication nonadherence among older adults: findings from a nationally representative sample. J Am Geriatr Soc 2019;67:2463-73.

28 Jan S, Laba T-L, Essue BM, et al. Action to address the household economic burden of non-communicable diseases. Lancet 2018;391:2047-58.

29 Lim JNW, Potrata B, Simonella L, et al. Barriers to early presentation of self-discovered breast cancer in Singapore and Malaysia: a qualitative multicentre study. BMJ Open 2015;5:e009863.

30 Ruddy K, Mayer E, Partridge A. Patient adherence and persistence with oral anticancer treatment. CA Cancer J Clin 2009;59:56-66.

31 Fenn KM, Evans SB, McCorkle R, et al. Impact of financial burden of cancer on survivors' quality of life. J Oncol Pract 2014;10:332-8.
32 Mongelli MN, Giri S, Peipert BJ, et al. Financial burden and quality of life among thyroid cancer survivors. Surgery 2020;167:631-7.

33 Jacob J, Palat G, Verghese N, et al. Health-related quality of life and its socio-economic and cultural predictors among advanced cancer patients: evidence from the approach cross-sectional survey in Hyderabad-India. BMC Palliat Care 2019;18:94.

34 Altice CK, Banegas MP, Tucker-Seeley RD, et al. Financial hardships experienced by cancer survivors: a systematic review. J Natl Cancer Inst 2017;109. doi:10.1093/jnci/djw205. [Epub ahead of print: 2010 2016].

35 van Dongen SI, de Nooijer K, Cramm JM, et al. Self-management of patients with advanced cancer: a systematic review of experiences and attitudes. Palliat Med 2020;34:160-78.

36 Cheng Q, Liu X, Li X, et al. Improving spiritual well-being among cancer patients: implications for clinical care. Support Care Cancer 2019;27:3403-9.

37 Wang C-W, Chow AY, Chan CL. The effects of life review interventions on spiritual well-being, psychological distress, and quality of life in patients with terminal or advanced cancer: a systematic review and meta-analysis of randomized controlled trials. Palliat Med 2017;31:883-94.

38 Lyckholm LJ, Coyne PJ, Kreutzer KO, et al. Barriers to effective palliative care for low-income patients in late stages of cancer: report of a study and strategies for defining and conquering the barriers. Nurs Clin North Am 2010;45:399-409.

39 Yeager KA, Sterk CE, Quest TE, et al. Managing one's symptoms: a qualitative study of low-income African Americans with advanced cancer. Cancer Nurs 2016;39:303-12.

40 Balboni T, Balboni M, Paulk ME, et al. Support of cancer patients' spiritual needs and associations with medical care costs at the end of life. Cancer 2011;117:5383-91.

41 Fitch MI, Sharp L, Hanly P, et al. Experiencing financial toxicity associated with cancer in publicly funded healthcare systems: a systematic review of qualitative studies. J Cancer Surviv 2021. doi:10.1007/s11764-021-01025-7. [Epub ahead of print: 15 Mar 2021].

42 Lockwood C, Munn Z, Porritt K. Qualitative research synthesis: methodological guidance for systematic reviewers utilizing metaaggregation. Int J Evid Based Healthc 2015;13:179-87.

43 Aromataris E, Briggs MZJ. Institute reviewer's manual. The Joanna Briggs Institute, 2017.

44 Pearson A. Balancing the evidence: incorporating the synthesis of qualitative data into systematic reviews. JBI Reports 2004;2:45-64.

45 Munn Z, Porritt K, Lockwood C, et al. Establishing confidence in the output of qualitative research synthesis: the ConQual approach. BMC Med Res Methodol 2014;14:108.

46 Tong A, Flemming K, Mclnnes E, et al. Enhancing transparency in reporting the synthesis of qualitative research: ENTREQ. BMC Med Res Methodol 2012;12:181. 